Document downloaded from:

http://hdl.handle.net/10251/60811

This paper must be cited as:

Vitale, R.; De Noord, OE.; Ferrer, A. (2014). A kernel-based approach for fault diagnosis in batch processes. Journal of Chemometrics. 28(8):697-707. doi:10.1002/cem.2629.

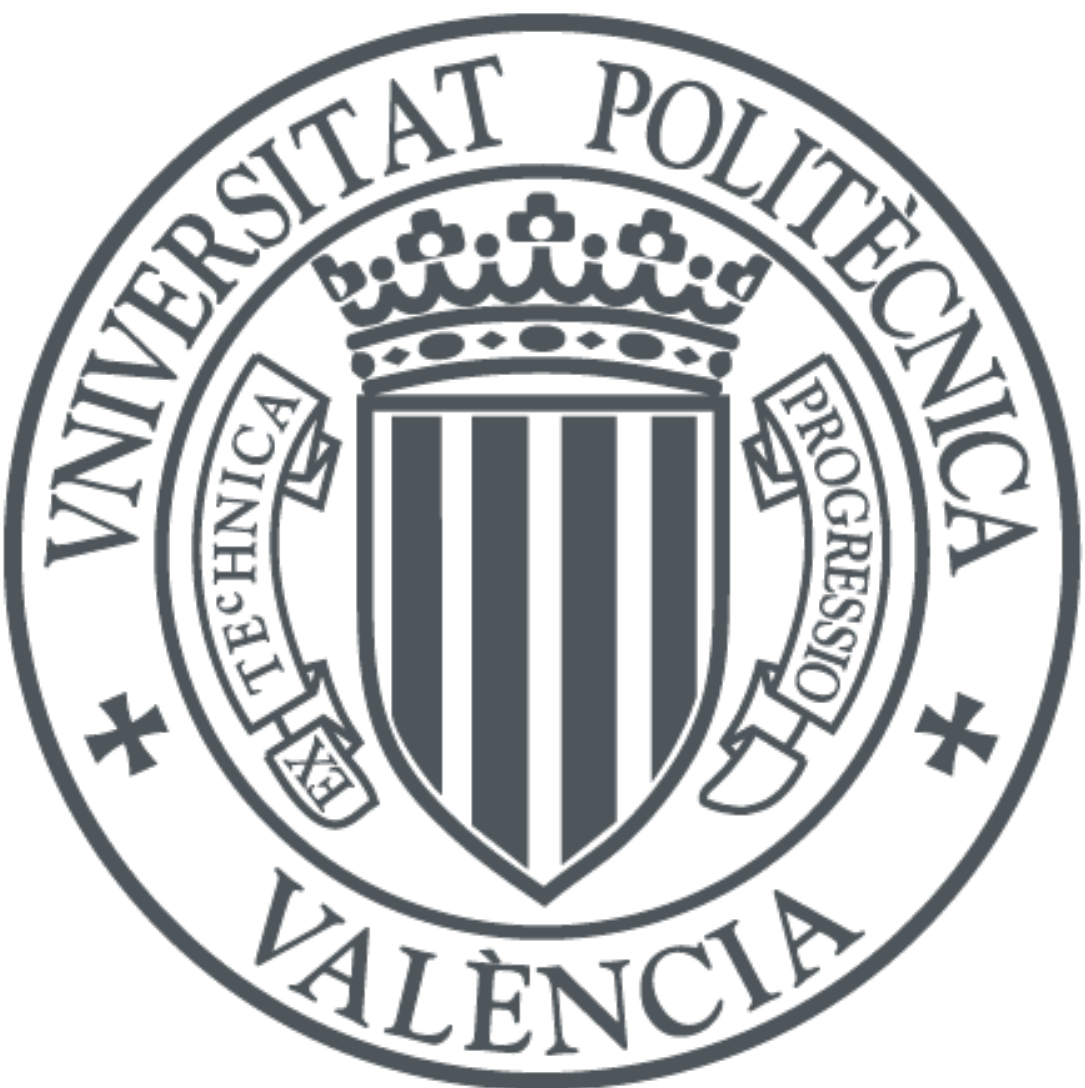

The final publication is available at

http://dx.doi.org/10.1002/cem.2629

Copyright Wiley

Additional Information 


\section{A kernel-based approach for fault diagnosis in batch processes}

2

3

4

5

6

7

8 Summary

9 This article explores the potential of kernel-based techniques for discriminating
R. Vitale ${ }^{a}$, O.E. de Noord ${ }^{b}$, A. Ferrer ${ }^{a}$

aDepartamento de Estadística e Investigación Operativa Aplicadas y Calidad, Universitat Politècnica de València, 46022, Valencia, Spain

bShell Global Solutions International B.V., Shell Technology Centre Amsterdam, PO Box 38000, 1030 BN Amsterdam, The Netherlands

\section{on- and off-specification batch runs, combining Kernel-PLSDA and three common} approaches to analyze batch data by means of bilinear models: Landmark Features Extraction, BatchWise Unfolding and VariableWise Unfolding. Gower's idea of pseudo-sample projection is exploited to recover the contribution of the initial variables to the final model and visualize those having the highest discriminant power. The results show the proposed approach provides an effective fault discrimination and enables a correct identification of the discriminant variables in the considered case studies.

Keywords: kernel-based methods, pseudo-sample projection, batch processes, fault discrimination, fault diagnosis.

\section{Introduction}

2 The presence of complex non-linear relationships in data may represent a difficult issue to solve when one tries to model them by means of the most common tools in chemometrics, such as Principal Component Analysis (PCA), Principal Component 
25 Regression (PCR) or Partial Least Squares Regression (PLSR). In fact, these 26 methods are not able to describe the underlying structure of datasets that are 27 affected by severe non-linearities, since they assume this structure is linear [1]. In 28 recent years, many techniques have been proposed to handle such kind of 29 situations: those based on non-linear PLS [2-3] and neural networks [4] have been 30 the most exploited ones. Unfortunately, these approaches often encompass many 31 adjustable parameters, are time and memory-consuming and may suffer from 32 overfitting and local minima. In order to avoid these issues, the so-called kernel 33 methods have been developed [5]. These techniques, which also comprehend 34 Support Vector Machines (SVM) [6], have been broadly used for solving non-linear 35 problems in chemistry [7-8], biology [9], informatics [10-11] and continuous 36 process chemometrics [12-13]. Their basic principle is common: before modeling 37 the data, a transformation of the original input space into a higher dimensional 38 one, the feature space, is performed by using specific kernel functions. This 39 permits to describe non-linear relationships in a linear form and to solve the 40 problem under study by means of classical linear methods. Hence, performing, for 41 instance, PCA, PLS or PLS Discriminant Analysis (PLSDA) after the data matrix 42 transformation results in Kernel-PCA (K-PCA), Kernel-PLS (K-PLS) and Kernel43 PLSDA (K-PLSDA), respectively. Unfortunately, the information about the weights 44 or the contributions of the original variables is lost. Different possibilities [8, 1445 16] to overcome this limitation exist, but authors often abstain from resorting to 46 them, since they do not permit to graphically visualize the relation between 47 variables and final models. Krooshof et al. [17] extended the idea of the non-linear 48 biplots, described by Gower and Hardings [18], to recover and visualize this 
49 specific information. In this case, the importance and influence of the variables is evaluated by constructing artificial samples, also known as pseudo-samples, whose

51 projection onto the space of the model gives information about their contribution

52 to it. This has been tested only on simulated datasets and in some metabolomic 53 studies [19-20].

54 The first aim of this article is to explore the potential of K-PLSDA for fault detection 55 in batch process analysis. Industrial batch processes generate massive amounts of 56 data, which are recorded for online treatment or posterior analysis. In particular, 57 during each batch run, $m=1 ; 2 ; \ldots ; M$ variables are measured at $t=1 ; 2 ; \ldots ; T$ time 58 points. Data collected for $i=1 ; 2 ; \ldots ; I$ batches are arranged in a three-way array $59(I \times M \times T)$. Even though techniques for directly modeling this structure exist, the 60 most widely used approach to extract exploitable information from this kind of data is to rearrange this three-way array into a matrix and then fit a bilinear model by means of one of the aforementioned chemometric tools [21]. The three most common unfolding strategies to perform this rearrangement are VariableWise Unfolding (VWU), BatchWise Unfolding (BWU) and Landmark Feature Extraction (LFE). VWU unfolds the original three-way array to a new matrix $(I T \times M)$ by

66 preserving the variable direction. BWU unfolds the initial structure to a new array

$67(I \times T M)$ by preserving the batch direction. LFE defines $F$ landmark features of the evolution of the $M$ variables in each batch and organizes them in a new matrix $(I \times F)$. A good survey of these techniques can be found in [22].

70 This article will be focused on the analysis of historical batch operations for the 71 troubleshooting of specific problems occurred during particular process runs. The 72 identification of the variables, which evolve differently with respect to an in- 
73 control situation, (the so-called fault diagnosis) is a key point in such cases.

74 Unfortunately, classical tools such as the contributions plots are useless if one 75 wants to appeal to kernel-based methods for batch process analysis, due to the 76 transformation of the original data matrix. For this reason, a new method based on 77 pseudo-sample projection is proposed here for recognizing those variables, which 78 deviates from the Normal Operation Conditions.

79

80 2. Materials and methods

$81 \quad 2.1$ Datasets

82 In this paper, three datasets are considered. The first is a simulated data array 83 containing the evolution of 10 variables at 25 sampling times in 30 different 84 batches: 15 are evolving under Normal Operation Conditions (NOC), while the 85 remaining 15 are faulty due to an increment in the variance of some variables. The 86 second one relates to a polymerization process described in [23] and consists of 23 87 batches (18 NOC and 5 off-specification) during which 10 variables are measured at 100 time points. In this case, both VWU and BWU were applied to the original three-way array. The third dataset was described in [24] and contains the values of

908 landmark features extracted from the variable trajectories of 71 batches (33 91 NOC, 10 on-specification but presenting an abnormally high quantity of residual 92 solvent, and 28 off-specification) of a pharmaceutical spray drying process. In 93 contrast with the original article, the second group of 10 batches was excluded 94 from the analysis in order to enable a simpler discrimination between on95 specification and off-specification runs, as for the previous datasets. 
98 The framework of the different kernel-based techniques is based on the so-called

99 kernel transformation, which is sketched in a general form in SI.1.

100 Its mathematical formulation is given by:

$$
K\left(\mathbf{x}_{i}, \mathbf{x}_{j}\right)=<\phi\left(\mathbf{x}_{i}\right), \phi\left(\mathbf{x}_{j}\right)>
$$

102 where $\mathbf{x}_{i}$ and $\mathbf{x}_{j}$ are two row vectors belonging to the original dataset, to which a 103 non-linear mapping function $\phi$ is applied, while $<$ and $>$ denote the inner product.

104 Therefore, the initial $N \times J$ data array, $\mathbf{X}$, where $N$ is the number of observations and $105 J$ the number of measured variables, is transformed into a new square symmetric $106 N \times N$ kernel matrix, $\mathbf{K}$, in which each position contains a value representing the 107 dissimilarity or distance between two different observations. When dealing with 108 kernel-based techniques, it is not necessary to know the mapping function a priori: 109 there are many generic kernel functions one can use in order to obtain $\mathbf{K}$ and all of 110 them exhibit two fundamental properties: i) they project the original data onto a 111 high dimensional space, the feature space; ii) they provide a way to calculate the 112 inner product between observations in this feature space.

113 The former permits to describe in a linear way possible non-linear relationships in 114 the data. The latter makes all the algorithms of the classical multivariate linear 115 techniques, which only use the inner product matrix (PCA, PLS and Fisher 116 Discriminant Analysis, FDA, as demonstrated by Cao et al. [1]), suitable for being 117 applied in the higher dimensional feature space. For the purposes of this article, 118 only three kernel transformations, the linear, the $2^{\text {nd }}$-order polynomial and the 119 Gaussian (executed by Radial Basis Functions) will be taken into account. Their 
mathematical formulations are listed in Table 1, together with the symbols of their

121 possible adjustable parameters.

\section{[INSERT HERE TABLE 1]}

\subsection{Pseudo-sample projection}

125 A pseudo-sample corresponds to a particular observation that carries all the 126 weight in one single variable. For example, the vector $[0,0, \ldots, 1,0, \ldots, 0]$,

127 represents one of the possible pseudo-samples associated to the variable $x_{j}$ of a 128 specific dataset. By projecting an observation like this onto the latent structure of a 129 classical 1-LV PLSDA model, the score for this new sample is calculated as follows:

$$
t_{\text {new }}=[0,0, \ldots, 1,0, \ldots, 0] \mathbf{w}^{*}=w_{j}
$$

131 This score is equal to the $j$-th value of the weighting vector, $\mathbf{w}^{*}$, and, thus, gives 132 information about the contribution of variable $x_{j}$ to the model. Creating for each 133 variable a pseudo-sample matrix, $\mathbf{P}_{j}$, which contains in the $j$-th column values 134 ranging from the minimum to the maximum of that variable and 0 in all the other entries, and projecting it onto the latent space, trajectories of points are constructed according to the equation:

$$
\mathbf{P}_{j} \mathbf{w}^{*}=\left[\begin{array}{c}
0, \ldots, 0, \min \left(\mathrm{x}_{j}\right), 0, \ldots, 0 \\
\ldots \\
\ldots \\
0, \ldots, 0, \max \left(\mathrm{x}_{j}\right), 0, \ldots, 0
\end{array}\right] \mathbf{w}^{*}=\left[\begin{array}{c}
\min \left(\mathrm{x}_{j}\right) w_{j} \\
\ldots \\
\ldots \\
\max \left(\mathrm{x}_{j}\right) w_{j}
\end{array}\right]
$$

138 It is straightforward to generalize this result to the case in which more than $1 \mathrm{LV}$ is

139 considered. Here, the matrix resulting from the previous operation defines the 140 geometrical locus of all the points lying along the direction determined by the 141 origin of the latent space and the point whose coordinates are defined by the 
142 weights of the $j$-th variable on the $A$ calculated LVs. In a classical PLSDA model,

143 representing these points does not provide any additional information, but, as will

144 be shown later, it is possible to get an idea from this kind of plot about how the

145 original variable evolves in the latent space when kernel-based methods are

146 applied. In fact, Postma et al. [19] demonstrated pseudo-sample projection permits

147 to recover the information related to the contribution of the original variables

148 when dealing with a Euclidean distance matrix, say D. In addition, D (double-

149 centered) is directly generated applying a linear kernel transformation to a generic

150 mean-centered dataset (see Appendix I for the details). Thus, it is possible to resort

151 to this strategy even when one uses K-PLSDA. In this case, it is only needed to

152 transform each pseudo-sample array into a pseudo-sample kernel one by the same

153 transformation as for the matrix used for constructing the model. The result is a

$154 P \times N$ array, which contains information about the dissimilarity between the $P$

155 pseudo-samples and the $N$ observations of the training set. The mathematical

156 formulation of this extension is described in Appendix II. Moreover, this is valid

157 not only in case one is exploiting a linear function to transform the analyzed data.

158 The pseudo-sample projection can be used when dealing with all the kernel

159 transformations, provided that they generate sets of distances which may be

160 embedded in a Euclidean space [18].

161 The whole procedure used in this article for building kernel-based models and

162 recovering the information about the influence of the original variables comprises

163 the following steps: i) Autoscale the original data matrix, $\mathbf{X}$; ii) Transform the

164 autoscaled dataset into a kernel matrix, K, by a specific kernel function; iii) Double-

165 center $\mathbf{K}$ so that: 


$$
\mathbf{K}_{c}=\mathbf{K}-\overline{\mathbf{K}}_{j}-\overline{\mathbf{K}}_{n}+\overline{\mathbf{K}}_{n j}
$$

167 where $\overline{\mathbf{K}}_{j}, \overline{\mathbf{K}}_{n}$ and $\overline{\mathbf{K}}_{n j}$ contain the column means, the row means and the overall

168 mean of the $\mathbf{K}$ matrix, respectively; iv) Fit a PLSDA model on $\mathbf{K}_{c} ; \mathrm{v}$ ) Create a

169 pseudo-sample matrix, $\mathbf{P}_{j}$, for each one of the original $J$ variables as described

170 before; vi) Apply to each pseudo-sample matrix the same kernel transformation as

171 for the training data in order to obtain a pseudo-sample kernel matrix, $\mathbf{P}_{j}^{\mathbf{K}}$; vii)

172 Double-center every $\mathbf{P}_{j}^{\mathbf{K}}$ so that:

$$
\mathbf{P}_{j c}^{\mathbf{K}}=\mathbf{P}_{j}^{\mathbf{K}}-\overline{\mathbf{K}}_{j}-\overline{\mathbf{P}}_{j p}{ }^{\mathbf{K}}+\overline{\mathbf{K}}_{n j}
$$

174 where the $p$-th row of $\overline{\mathbf{P}}_{j p}{ }^{\mathbf{K}}$ contains the mean of the $p$-th row of $\mathbf{P}_{j}^{\mathbf{K}}$. Notice that $\overline{\mathbf{K}}_{n}$

175 is substituted by the term $\overline{\mathbf{P}}_{j p}{ }^{\mathbf{K}}$ since the total number of rows of $\mathbf{P}_{j}^{\mathbf{K}}$ is usually 176 different from the number of rows of $\mathbf{K}$; viii) Project each $j$-th pseudo-sample 177 kernel matrix onto the latent structure, as follows

$$
\mathbf{T}_{j, p s}=\mathbf{P}_{j c}^{\mathbf{K}} \mathbf{W}^{* \mathbf{K}}
$$

179 where $\mathbf{W}^{* \mathrm{~K}}$ corresponds to the weighting matrix of the K-PLSDA model; ix) Plot the 180 predicted scores, $\mathbf{T}_{j, p s}$, for recovering the information about the contribution of 181 each original variable to the K-PLSDA model.

182

\section{3. Results and discussion}

\subsection{Simulated example}

185 A 750x2 set of scores following trimmed circular trajectories was generated, 186 creating 2 classes of 15 different trajectories of 25 observations each, as shown in 187 Figure 1. 
189 Every trimmed circular profile represents a proper batch score trajectory, which 190 defines its evolution in the latent variable space and might have been obtained 191 applying PCA on a VWU data array. So, multiplying this set of scores by a $2 \times 10$ 192 transposed matrix of loadings, calculated building a PCA model on real process 193 data, a 750x10 dataset was constructed, which contains the evolution of 10 194 variables at 25 sampling times in 30 different runs. As shown in Figure 2, this 195 results in two classes of batches characterized by differences in the variance of the 196 measured variables and not in their mean values (e.g. due to sensor or controller 197 faults).

[INSERT HERE FIGURE 2]

199 To verify whether pseudo-sample projection enables the correct identification of 200 the discriminant variables, three columns of the resulting dataset were substituted 201 by three white noise vectors.

202 Finally, the whole array was divided into a training and a test set, containing 500 203 (20 complete batches) and 250 (10 complete batches) observations, respectively. 204 Batch selection was randomly performed class-wise.

205 Four different cross-validated classification models, with a growing degree of non206 linearity, were built on the simulated data. The performance of the final models is 207 summarized in Table 2.

[INSERT HERE TABLE 2]

209 Clearly, the two classes cannot be satisfactorily separated by classical PLSDA and 210 K-PLSDA with a linear kernel transformation. However, resorting to non-linear 211 kernel functions permits to correctly discriminate most of the observations 212 belonging to the two different categories for both training and test set. The best 
213 correct classification rate is obtained by the $2^{\text {nd }}$-order polynomial kernel model,

214 whose scores and predicted class values plots are shown in Figure 3.

216 Here, it is important to take into account that each one of the represented symbols 217 corresponds to a specific time point of a particular batch. K-PLSDA is then able to 218 correctly discriminate most of the time samples in which the process is 219 progressing under Normal Operation Conditions or not.

220 The highest discrimination ability of this model is reasonable, considering that the 221 differences between the two classes under study are associated to the variance of

222 the measured variables, which results, indeed, in a quadratic transformation of the 223 original data.

224 In order to check whether pseudo-sample projection permits to recover the 225 information about the discriminant power of the original variables, for each 226 column of the simulated data matrix, a $20 \times 10$ pseudo-sample array was built, 227 transformed and projected onto the model space as described in Section 2.3. 228 Figure 4 shows the obtained outcomes.

\section{[INSERT HERE FIGURE 4]}

230 The different trajectories represent the predicted scores calculated from the 231 pseudo-sample kernel matrices constructed for all the original variables 232 (numbered from 1 to 10 ). The blue dotted line corresponds to the discriminant 233 direction between the centers of gravity of the two classes of observations, 234 obtained from Figure 3a. The font-size of the numerical characters constituting 235 each trajectory increases in correspondence of regions of the latent space where 236 the respective variables assume higher values and viceversa. So, comparing this 
237 graph with the scores plot in Figure 3a, it is rather clear that the second class (red 238 squares) contains batch runs associated to either higher or lower values of the 239 labeled variables than those belonging to the first group (blue dots).

240 In order to define an objective criterion for evaluating the discriminant power of 241 the original variables, the cosine of the angle formed by the blue dotted line and 242 each trajectory was calculated. These values are listed in Table 3 and clearly 243 indicate that all the variables except $x_{8}, x_{9}$ and $x_{10}$ have good discriminant power 244 (i.e. angle cosines close to 1). Notice that for these latter there are no clear 245 trajectories (see Figure 4) and then the cosine of the angles cannot be precisely 246 calculated. This is coherent with the simulated data shown in Figure 2 where the 247 variables with differences in variance between on- and off-specification batches 248 are $x_{1}$ to $x_{7}$.

\section{[INSERT HERE TABLE 3]}

\section{$250 \quad 3.2 \mathrm{VWU} / \mathrm{K}$-PLSDA (polymerization process)}

251 The polymerization dataset under consideration contains observations related to 252 on- and off-specification batches, but the time period in which their evolution 253 differs is unknown. In order to identify it, a preliminary exploratory K-PCA model 254 was built, using a linear kernel transformation, on all the NOC process runs. 255 Hotelling's T² and SPE (Squared Prediction Error) statistics were calculated for the 256 remaining faulty ones after their projection onto the latent variable space. The 257 resulting $\mathrm{T}^{2}$ and SPE control charts are shown in SI.2.

258 It is straightforward to identify that the initial time interval of the process (the first 25915 time points) is where the off-specification batches have different evolution than 260 the on-specification ones. In this case, using classical PCA instead of K-PCA would 
261 have returned very similar results (not shown). Therefore, in order to discriminate

262 the two classes, only this period was considered in the following step of data 263 analysis. So, the initial VWU matrix was reduced to a $345 \times 10$ one, which was then 264 divided into a training and a test set, containing 225 and 120 observations, 265 respectively. Their selection was performed leaving outside the training set all the 266 time samples associated to 6 on-specification and 2 off-specification batches, 267 randomly chosen. A linear kernel transformation was applied to the calibration 268 data and a cross-validated 2-LV PLSDA model was built on the resulting $225 \times 225$ 269 kernel matrix. Its performance was evaluated in terms of $\mathrm{R}^{2}$ and $\mathrm{Q}^{2}$, showing 270 values of $94.7 \%$ and $94.3 \%$, respectively. In order to assess its prediction ability 271 the observations of the test set were transformed in the same way as those of the 272 training set (generating a kernel test matrix with dimension $120 \times 225$ ), projected 273 onto its latent structure and, according to their predicted $y$ values, assigned to one 274 of the two considered classes. Results are displayed in Figure 5. [INSERT HERE FIGURE 5]

276 The K-PLSDA scores plot on the two latent variables (Figure 5a) shows a perfect 277 separation between the observations belonging to the different categories. Since 278 the model was built after the transformation of a VWU data matrix, as for the 279 previous case, each represented symbol corresponds to a specific time point of a 280 particular batch. As will be shown, this is a fundamental difference with respect to 281 the other described approaches based on BWU and LFE.

282 The good discrimination is corroborated by the plot of the predicted class values 283 (Figure 5b). 100\% correct classification rate is obtained both in training and test 284 sets for the two categories. As aforementioned, plotting directly the loadings or the 
285 weights of models like this is totally uninformative since the kernel matrix only 286 contains values of dissimilarity between observations. This is the reason why 287 pseudo-sample projection is needed to recover the information about the 288 contribution of the original variables to the discrimination between the two 289 classes. For each column of the VWU data matrix, a $20 \times 10$ pseudo-sample array 290 was built, transformed and projected onto the model space. Figure 6 shows the 291 obtained outcomes.

293 The values of the cosine of the angle formed by the blue dotted line and each 294 trajectory are summarized in Table 4.

[INSERT HERE TABLE 4]

296 Variables $x_{4}, x_{7}, x_{9}$ and $x_{10}$ are proved to be the most significant ones with values of 297 this cosine clearly higher than the other ones.

298 Also in this case, the font-size of the numerical characters of each trajectory 299 increases in correspondence to regions of the latent space where the respective 300 variables assume higher values and viceversa. Therefore, comparing this graph 301 with the scores plot in Figure 5a, it is possible to infer that off-specification batches 302 are characterized by higher values of variables $x_{7}, X_{9}$ and $x_{10}$ and lower values of 303 variable $x_{4}$ in comparison to the on-specification ones, as confirmed by 304 representing their original temporal evolution, shown in SI.3.

305 Similar results are obtained from a PLSDA model without a kernel transformation, 306 as highlighted in SI.4. The plot is associated to a specific time point of the interval 307 during which the off-specification batches evolve differently from the others, but 308 the displayed profile is consistent with all the other analyzed time samples. 
310 The same procedure described in Section 2.3 was then applied to the BWU data 311 matrix $(23 \times 1000)$ from the polymerization process. A linear kernel was chosen for 312 the transformation of the original array. Since only few observations (i.e. batches) 313 were available for the calibration of the model, it was not possible to evaluate its 314 predictive ability via an external test set. As will be discussed later, permutation 315 tests were used for overcoming this limitation. PLSDA was applied on the resulting $31623 \times 23$ kernel matrix. Results are shown in Figure 7 . [INSERT HERE FIGURE 7]

318 The final Leave-One-Out CV 2-LV model shows $\mathrm{R}^{2}$ and $\mathrm{Q}^{2}$ values of $97.5 \%$ and $31995.9 \%$, respectively. The separation of the two classes is perfect, leading to $100 \%$ 320 correct classification rate both in calibration and cross-validation. Unlike the VWU 321 case, here each represented symbol corresponds to a whole batch: therefore, the 322 discrimination highlights the difference between on- and off-specification batches. 323 Due to the structure of the original dataset, 1000 pseudo-sample trajectories 324 showing the importance of a particular variable measured at a specific time spot 325 were constructed, each one constituted by 20 points. Representing all these 326 trajectories would have made the plot uninterpretable. For this reason, only those 327 related to the time period, during which the difference in the evolution of the 328 batches was detected, according to the initial K-PCA analysis discussed in Section 329 3.2, were included in SI.5. The graph is divided in 10 sections as the number of 330 original variables. Inside every section, the pseudo-sample trajectories for the respective variable at the different considered time points are represented. The

332 blue dotted line corresponds to the class discriminant direction. As in the VWU 
333 case, the cosine of the angle formed by each trajectory and this direction was 334 selected as criterion of variable importance. In SI.5, only the pseudo-samples 335 trajectories characterized by a value of the amplitude of this angle lower than $30^{\circ}$ 336 are black-coloured. Variables $x_{4}, x_{9}$ and $x_{10}$ are found to have high contributions to 337 the model approximately for the whole interval under study, while variable $x_{7}$ is 338 significant only in part of this period. This is also shown by plotting the values of 339 the cosine of the angles formed by the series of respective trajectories and the 340 discriminant direction with respect to the batch time, as illustrated in Figure 8. [INSERT HERE FIGURE 8]

342 Variables $x_{2}, x_{5}, x_{6}$ also proved to have high significance in small periods. In such 343 cases, a further investigation of the original variable trajectories is always needed 344 to properly identify the root causes generating problems during the process.

345 As for the previous examples, the pseudo-sample plot (SI.5) is built using larger 346 bullet-size in correspondence of the zones of the latent space in which the 347 respective variable assumes higher values and viceversa. Hence, it is 348 straightforward to conclude that on-specification batches are characterized by 349 lower values of variables $x_{7}, x_{9}$ and $x_{10}$ and higher values of variable $x_{4}$ in 350 comparison to the off-specification runs, which exactly corresponds to the 351 outcomes discussed before.

352 In order to validate the final model, a permutation test [26] was performed. SI.6 353 shows the validation plots obtained for the BWU kernel matrix. The difference 354 between the two categories under study is proved to be statistically significant ( $p$ 355 value $<\frac{1}{2000}=0.005$ for both $\mathrm{R}^{2}$ and $\mathrm{Q}^{2}$ ). 
357 A K-PLSDA model was built on the LFE data matrix $(61 \times 8)$ from the 358 pharmaceutical spray drying process. Among the initial observations, 12 (7 on359 specification and 5 off-specification) were found having abnormally high residuals 360 and therefore were excluded from the final classification in order not to jeopardize 361 its quality. A further K-PLSDA model was then constructed on the reduced LFE 362 dataset $(49 \times 8)$. Since a linear kernel transformation did not provide good results, a 363 Radial Basis Function was applied to the original array. The $\sigma$ parameter was 364 optimized by leave-one-out cross-validation and fixed at a value of 0.8 . Smaller 365 values would have generated over-fitting and hardly interpretable pseudo-sample 366 trajectories. A cross-validated 2-LV PLSDA model was built on the resulting $49 \times 49$ 367 kernel matrix. Its performance was assessed according to the values of $\mathrm{R}^{2}(73.8 \%)$ 368 and $\mathrm{Q}^{2}(43.6 \%)$. Figure 9 displays the K-PLSDA scores plot and the predicted $y$ 369 values for all the observations in calibration and cross-validation. [INSERT HERE FIGURE 9]

371 Also in this case, each represented symbol corresponds to a whole process run. 372 Here, the model does not guarantee high performance as those described in the 373 previous examples. This is due to the fact that the selected landmark features have 374 quite low correlation to the quality of the batches [24]. Nevertheless, resorting to a 375 K-PLSDA model enabled a satisfying discrimination even dealing with a dataset 376 like this $(73.08 \%$ and $91.30 \%$ correct classification rate in cross-validation for the 377 two categories, respectively). In order to recover the information about the 378 original variables, a $20 \times 8$ pseudo-sample matrix was constructed per each column 379 of the initial LFE array. The resulting pseudo-sample trajectories are represented 380 in Figure 10. 
382 The graph is built in the same way as SI.5. As stated by Gower [18], non-linear 383 kernel transformations lead to non-linear pseudo-sample trajectories. Since it is 384 impossible to univocally define an angle between the separation direction and a 385 curved line, the interpretation of the variable importance is not straightforward. 386 On the other hand, by inspecting the plot, it is rather clear that the only variables, 387 whose pseudo-sample evolution is correlated to the blue dotted line, are $x_{1}, x_{2}$ and $388 x_{8}$. All the other trajectories cover circular paths (variables $x_{4}, x_{5}$ and $x_{6}$ ) or show a 389 nearly linear trend with a direction almost orthogonal to the discriminant one 390 (variable $x_{3}$ and $x_{7}$ ). As in the previous cases, larger font-sizes indicate regions of 391 the latent space in which the labeled variables assume higher values. So, it is easy 392 to infer off-specification batches are characterized by lower values of variable $x_{8}$ 393 and by higher values of variables $x_{1}$ and $x_{2}$. The obtained outcomes are coherent 394 with the conclusions reached in the original article by García-Muñoz et al. [24], 395 where it is detailed "a high-quality product is also associated with low solvent level 396 in the collector tank (variable $x_{1}$ )", "batches that progress faster (with higher 397 values of $x_{8}$ ) tend to be those with high product quality" and "a low temperature in 398 the dryer at the end of stage 1 (variable $x_{2}$ ) might also seem desirable".

399 For assessing the model performance, a permutation test was executed, due to the 400 small number of observations constituting the dataset. The results are shown in 401 SI.7.

402 The model is found to be statistically significant with respect to the other 403 permuted classifications $\left(\mathrm{R}^{2} p\right.$-value $=0.003, \mathrm{Q}^{2} p$-value $\left.<0.005\right)$. However, even if 404 the $\mathrm{Q}^{2}$ of the final model is always larger than those calculated modifying the class 
405 label of the single observations, its $\mathrm{R}^{2}$ is lower than some obtained after the class 406 randomization. This aspect might be a caution indicator of the presence of 407 variables whose contribution is unrelated to the class of the objects [27]. This issue 408 is quite common when dealing with LFE [22]. In general, selecting a set of 409 landmark features, which summarize the evolution and the differences between 410 on-specification and off-specification batches in a proper way, may not be obvious: 411 this may often lead to less reliable results when dealing with such kind of datasets 412 than directly operating on the evolution of the measured variables during time.

\section{4. Comparison between K-PLSDA and classical PLSDA models}

415 The analysis of the simulated dataset highlighted the main advantage of using non416 linear kernel-based classification methods over classical PLSDA. In fact, when 417 complex data structures have to be modeled, such bilinear technique leads to low 418 and unsatisfactory correct classification rates, which jeopardizes the fault 419 detection. In such cases, exploiting non-linear classifiers radically improves the 420 quality of the discrimination and the identification of the process runs, which did 421 not progress under Normal Operation Conditions. This is also confirmed by the 422 results obtained in the second case study. In fact, for the first real dataset, for both 423 the VWU and BWU matrices, resorting to K-PLSDA for discriminating NOC batches 424 from faulty ones did not result in significantly better performance than building a 425 classical PLSDA model (results not shown). This similarity is a consequence of the 426 fact that a linear kernel transformation permitted to obtain satisfying correct 427 classification rates for the two considered classes, which means the original data 428 were not affected by strongly non-linear relationships [1] and, therefore, they 
429 might have been analyzed by means of conventional bilinear approaches, obtaining 430 very similar outcomes.

431 On the other hand, when the LFE matrix was dealt with for the second real dataset,

432 the best discrimination between the two categories under study was obtained by a 433 kernel transformation performed using a radial-basis function (RBF). Here, if one 434 compares the RBF K-PLSDA scores and $y$-predicted plots, displayed in Figure 9, 435 with the ones constructed when a classical PLSDA model is built on the original 436 matrix, shown in Figure 11, it is possible to verify the clear improvement in the 437 separation between the observations belonging to the different classes, achieved 438 when the kernel-based method is applied. [INSERT HERE FIGURE 11]

\section{$441 \quad$ 5. Conclusions}

442 In this article, a novel approach for fault discrimination and diagnosis in batch 443 processes was proposed. It combines the ability of kernel-based classification 444 techniques (in particular K-PLSDA) of dealing with complex non-linear data 445 structures with the power of pseudo-sample projection (originally conceived by 446 John Gower) for recovering the information related to the contribution of the 447 initial variables to the final model, which permits to overcome one of the main 448 drawbacks of these methods.

449 K-PLSDA shows similar performance to classical PLSDA, when linear 450 transformations are appropriate for the datasets under study, but leads to better 451 discrimination between the classes in case non-linear functions are needed for 452 modelling more complex data structures, as clearly highlighted by the analysis of 
453 the simulated and LFE datasets. In both scenarios, the pseudo-sample projection

454 enables a correct identification of the discriminant variables. For all these reasons,

455 the authors' suggestion for practical users is to resort to non-linear K-PLSDA when

456 standard bilinear techniques provide unsatisfactory outcomes.

457 Moreover, it was seen that the described strategy may constitute a powerful

458 method for detecting differences in the variance of the variable trajectories

459 measured during batch runs and then could represent an important crossroad in

460 this specific field of statistical process monitoring and control.

461 These satisfying results can be certainly considered a good starting point for

462 implementing this strategy as a complementary tool for Batch Multivariate

463 Statistical Process Control (BMSPC) methods.

464

465 Appendix I

466 Relationship between the Euclidean Distance Matrix, D, and the inner product 467 matrix, $\mathbf{X X}^{\mathrm{T}}$

468 The Euclidean distance between two observations contained in a generic data 469 matrix $\mathbf{X}_{(N \times M)}, \mathbf{x}_{i}$ and $\mathbf{x}_{j}$, is:

$$
d_{i, j}=\left\|\mathbf{x}_{i}-\mathbf{x}_{j}\right\|^{2}=\left(\mathbf{x}_{i}-\mathbf{x}_{j}\right)^{\mathrm{T}}\left(\mathbf{x}_{i}-\mathbf{x}_{j}\right)=\mathbf{x}_{i}^{\mathrm{T}} \mathbf{x}_{i}+\mathbf{x}_{j}^{\mathrm{T}} \mathbf{x}_{j}-2 \mathbf{x}_{i}^{\mathrm{T}} \mathbf{x}_{j}
$$

471 Let $\mathbf{F}$ be the inner product matrix so that:

$$
\mathbf{F}=\mathbf{X} \mathbf{X}^{\mathrm{T}}
$$

473 The Euclidean distance matrix is then defined as:

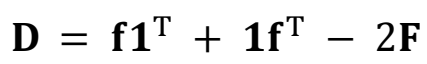

475 where $\mathbf{f}=\operatorname{diag}(\mathbf{F})$ and $\mathbf{1}=(1,1, \ldots, 1)^{\mathrm{T}}$. Centering $\mathbf{X}$ so that: 
477 it is obtained:

478

$$
\widetilde{\mathbf{F}}=\widetilde{\mathbf{X}} \widetilde{\mathbf{X}}^{\mathrm{T}}=
$$

479

$$
\left(\mathbf{X}-\frac{1}{n} \mathbf{1 1}^{\mathrm{T}} \mathbf{X}\right)\left(\mathbf{X}-\frac{1}{n} \mathbf{1 1}^{\mathrm{T}} \mathbf{X}\right)^{\mathrm{T}}=\mathbf{F}-\frac{1}{n} \mathbf{F} \mathbf{1 1}{ }^{\mathrm{T}}-\frac{1}{n} \mathbf{1 1}^{\mathrm{T}} \mathbf{F}+\frac{1}{n^{2}} \mathbf{1 1}^{\mathrm{T}} \mathbf{F} \mathbf{1 1}{ }^{\mathrm{T}}
$$

480 Consider the double-centered Euclidean distance matrix:

481

$$
\mathbf{B}=-\frac{1}{2} \mathbf{H D H}^{\mathrm{T}}
$$

482 where $\mathbf{H}=\mathbf{I}-\frac{1}{n} \mathbf{1 1}^{\mathrm{T}}$. So:

483

$$
\mathbf{B}=-\frac{1}{2} \mathbf{H}\left(\mathbf{f} \mathbf{1}^{\mathrm{T}}+\mathbf{1} \mathbf{f}^{\mathrm{T}}-2 \mathbf{F}\right) \mathbf{H}^{\mathrm{T}}
$$

484 Since:

485

$$
\mathbf{f} 1^{\mathrm{T}} \mathbf{H}^{\mathrm{T}}=\mathbf{f} \mathbf{1}^{\mathrm{T}}\left(\mathbf{I}-\frac{1}{n} \mathbf{1} \mathbf{1}^{\mathrm{T}}\right)^{\mathrm{T}}=\mathbf{f} \mathbf{1}^{\mathrm{T}}-\mathbf{f}\left(\frac{\mathbf{1}^{\mathrm{T}} \mathbf{1}}{n}\right) \mathbf{1}^{\mathrm{T}}=0
$$

486 it is verified:

487

$$
\mathbf{H} \mathbf{f} \mathbf{1}^{\mathrm{T}} \mathbf{H}^{\mathrm{T}}=0=\mathbf{H} \mathbf{1} \mathbf{f}^{\mathrm{T}} \mathbf{H}^{\mathrm{T}}
$$

488

489

$$
\mathbf{B}=\mathbf{H F} \mathbf{H}^{\mathrm{T}}=\left(\mathbf{I}-\frac{1}{n} \mathbf{1 1}^{\mathrm{T}}\right) \mathbf{F}\left(\mathbf{I}-\frac{1}{n} \mathbf{1 1}^{\mathrm{T}}\right)^{\mathrm{T}}=\mathbf{F}-\frac{1}{n} \mathbf{F} 11^{\mathrm{T}}-\frac{1}{n} \mathbf{1 1}^{\mathrm{T}} \mathbf{F}+
$$

490

$$
\frac{1}{n^{2}} \mathbf{1}\left(\mathbf{1}^{\mathrm{T}} \mathbf{F} \mathbf{1}\right) \mathbf{1}^{\mathrm{T}}=\tilde{\mathbf{F}}
$$

491 that is:

492

$$
\mathbf{B}=-\frac{1}{2} \mathbf{H D H}^{\mathrm{T}}=\widetilde{\mathbf{X}} \widetilde{\mathbf{X}}^{\mathrm{T}}
$$

493 The Euclidean distance matrix $\mathbf{D}$ after double-centering is equal to the inner 494 product matrix $\widetilde{\mathbf{X}} \widetilde{\mathbf{X}}^{\mathrm{T}}$.

495

\section{Appendix II}

497 Extension of the pseudo-samples projection to the feature space 
498 Suppose one has built a 1-LV PLSDA model on a double-centered Euclidean 499 distance matrix, $\mathbf{B}_{(N \times N)}$, obtained based on the distances between the observations 500 in $\mathbf{X}_{(N \times M)}$. The scores of the objects belonging to the training set are calculated as:

$$
\mathbf{t}_{(N \times 1)}=\mathbf{B}_{(N \times N)} \mathbf{w}^{* \mathbf{B}}(N \times 1)
$$

502 where $\mathbf{w}^{* \mathbf{B}}$ represents the weighting vector obtained from $\mathbf{B}$, which does not show 503 the contribution of the $M$ original variables. Substituting (17) in (18):

$$
\mathbf{t}_{(n \times 1)}=\widetilde{\mathbf{X}}_{(N \times M)} \widetilde{\mathbf{X}}_{(M \times N)}^{\mathrm{T}} \mathbf{W}^{*} \mathbf{B}_{(N \times 1)}
$$

505 Rewriting formula (18):

506

$$
\mathbf{t}_{(N \times 1)}=\widetilde{\mathbf{X}}_{(N \times M)}\left(\widetilde{\mathbf{X}}^{\mathrm{T}}{ }_{(M \times N)} \mathbf{w}^{\left.* \mathbf{B}_{(N \times 1)}\right)}=\widetilde{\mathbf{X}}_{(N \times M)} \mathbf{w}_{(M \times 1)}^{* \prime}\right.
$$

507 where $\mathbf{w}^{* \prime}$ actually contains information about the influence of the $M$ original 508 variables on the model.

509 Projecting the pseudo-sample $[0, \ldots, 0,1,0, \ldots, 0]_{(1 \times M)}$ onto the PLSDA latent 510 structure and calculating the respective predicted score results in:

$$
t_{n e w}=[0, \ldots, 0,1,0, \ldots, 0]_{(1 \times M)} \widetilde{\mathbf{X}}_{(M \times N)}^{\mathrm{T}} \mathbf{w}^{* \mathbf{B}_{(N \times 1)}}=[0, \ldots, 0,1,0, \ldots, 0]_{(1 \times M)} \mathbf{w}_{(M \times 1)}
$$

$$
=w_{j}^{* \prime}
$$

$513 t_{n e w}$ is exactly equal to the $j$-th value of the vector $\mathbf{w}^{* \prime}$. When using a series of 514 pseudo-samples instead of only one, trajectories of points are constructed, whose 515 evolution gives an idea about how the original variables contribute to the final 516 model. Since the linear kernel matrix exactly corresponds to the inner product one, 517 it is straightforward to infer this outcome is valid when dealing with the respective 518 data transformation. However, as stated by Gower [18], the same property is 519 verified when dealing with all those generating sets of distances, which may be 520 embedded in a Euclidean space. The Euclidean nature of the Gaussian kernel is 521 particularly clear since it is calculated as a function of the Euclidean distance [28]. 
522

523

524

525

526

527

528

529

530

531

532

533

534

535

536

537

538

539

540

541

542

543

544

545

\section{References}

[1] Cao D, Liang Y, Xu Q, Hu Q, Zhang L, Fu G. Exploring nonlinear relationships in chemical data using kernel-based methods. Chemometr. Intell. Lab. 2011; 107:106-115

[2] Walczak B, Massart D. The Radial Basis Functions - Partial Least Squares approach as a flexible non-linear regression technique. Anal. Chim. Acta 1996; 331:177-185

[3] Walczak B, Massart D. Application of Radial Basis Functions - Partial Least Squares to non-linear pattern recognition problems: diagnosis of process faults. Anal. Chim. Acta 1996; 331:187-193

[4] Gasteiger J, Zupan J. Neural Networks in Chemistry. Angew. Chem. Int. Ed. Engl. 1993; 32:503-527

[5] Schölkopf B, Smola A. Learning with Kernels (1st edn). MIT Press: Cambridge, UK, 2002

[6] Li H, Liang Y, Xu Q. Support vector machines and its applications in chemistry. Chemometr. Intell. Lab. 2009; 95:188-198

[7] Williams P. Influence of water on prediction of composition and quality factors: the Aquaphotomics of low moisture agricultural materials. J. Near Infrared Spectrosc. 2009; 17: 315-328

[8] Tan C, Li M. Mutual information-induced interval selection combined with kernel partial least squares for near-infrared spectral calibration. Spectrochim. Acta Pt. A-Mol. Biomol. Spectrosc. 2008; 71:1266-1273

[9] Embrechts M, Ekins S. Classification of Metabolites with Kernel-Partial Least Squares (K-PLS). Drug Metab. Dispos. 2007; 35:325-327 
546 [10] Arenas-Garcia J, Camps-Valls G. Efficient Kernel Orthonormalized PLS for

547 Remote Sensing Applications. IEEE Trans. Geosci. Remote Sens. 2008;

$548 \quad 46: 2872-2881$

549 [11] Struc V, Pavesic N. Gabor-based kernel partial-least-squares 550 discrimination features for face recognition. Informatica 2009; 20:115$551 \quad 138$

552 [12] Sun R, Tsung F. A kernel-distance-based multivariate control chart using $553 \quad$ support vector methods. Int. J. Prod. Res. 2003; 41:2975-2989

554 [13] Lee J, Yoo C, Choi S, Vanrolleghem P, Lee I. Nonlinear process monitoring 555 using kernel principal component analysis. Chem. Eng. Sci. 2004; 59:223234

[14] Bennett K, Embrechts M. Advances in Learning Theory: Methods, Models 558 and Applications (1st edn). IOS Press: Amsterdam, The Netherlands,

560 [15] Kewley R, Embrechts M, Breneman C. Data strip mining for the virtual 561 design of pharmaceuticals with neural networks. IEEE Trans. Neural Netw. 2000; 11:668-679

563 [16] Ūstūn B, Melssen W, Buydens L. Visualisation and interpretation of 564 Support Vector Regression models. Anal. Chim. Acta 2007; 595:299-309

565 [17] Krooshof P, Ūstūn B, Postma G, Buydens L. Visualization and Recovery of 566 the (Bio)chemical Interesting Variables in Data Analysis with Support 567 Vector Machine Classification. Anal. Chem. 2010; 82:7000-7007 568 [18] Gower J, Harding S. Nonlinear biplots. Biometrika 1988; 75:445-455 
[19] Postma G, Krooshof P, Buydens L. Opening the kernel of kernel partial least squares and support vector machines. Anal. Chim. Acta 2011; 705:123-134

[20] Smolinska A, Blanchet L, Coulier L, Ampt K, Luider T, Hintzen R, Wijmenga S, Buydens L. Interpretation and Visualization of Non-Linear Data Fusion in Kernel Space: Study on Metabolomic Characterization of Progression of Multiple Sclerosis. PLoS ONE 2012; 7:e38163

[21] Camacho J, Picó J, Ferrer A. Bilinear modelling of batch processes. Part I: theoretical discussion. J. Chemometr. 2008; 22:299-308

[27] Quintás G, Portillo N, García-Cañaveras J, Vicente Castell J, Ferrer A, Lahoz A. Chemometric approaches to improve PLSDA model outcome for 
predicting human non-alcoholic fatty liver disease using UPLC-MS as a metabolic profiling tool. Metabolomics 2012; 8:86-98

595 [28] Courrieu P. Straight monotonic embedding of data sets in Euclidean space. Neural Networks 2002; 15:1185-1196 


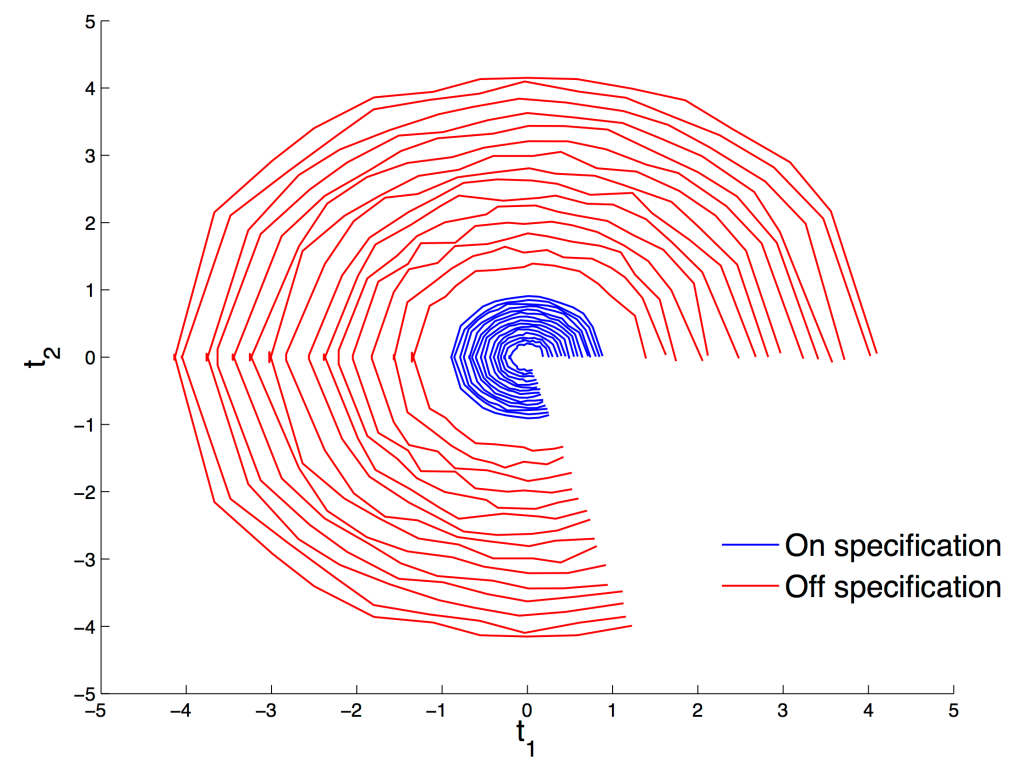

Figure 1: On-specification and off-specification simulated batch score trajectories 

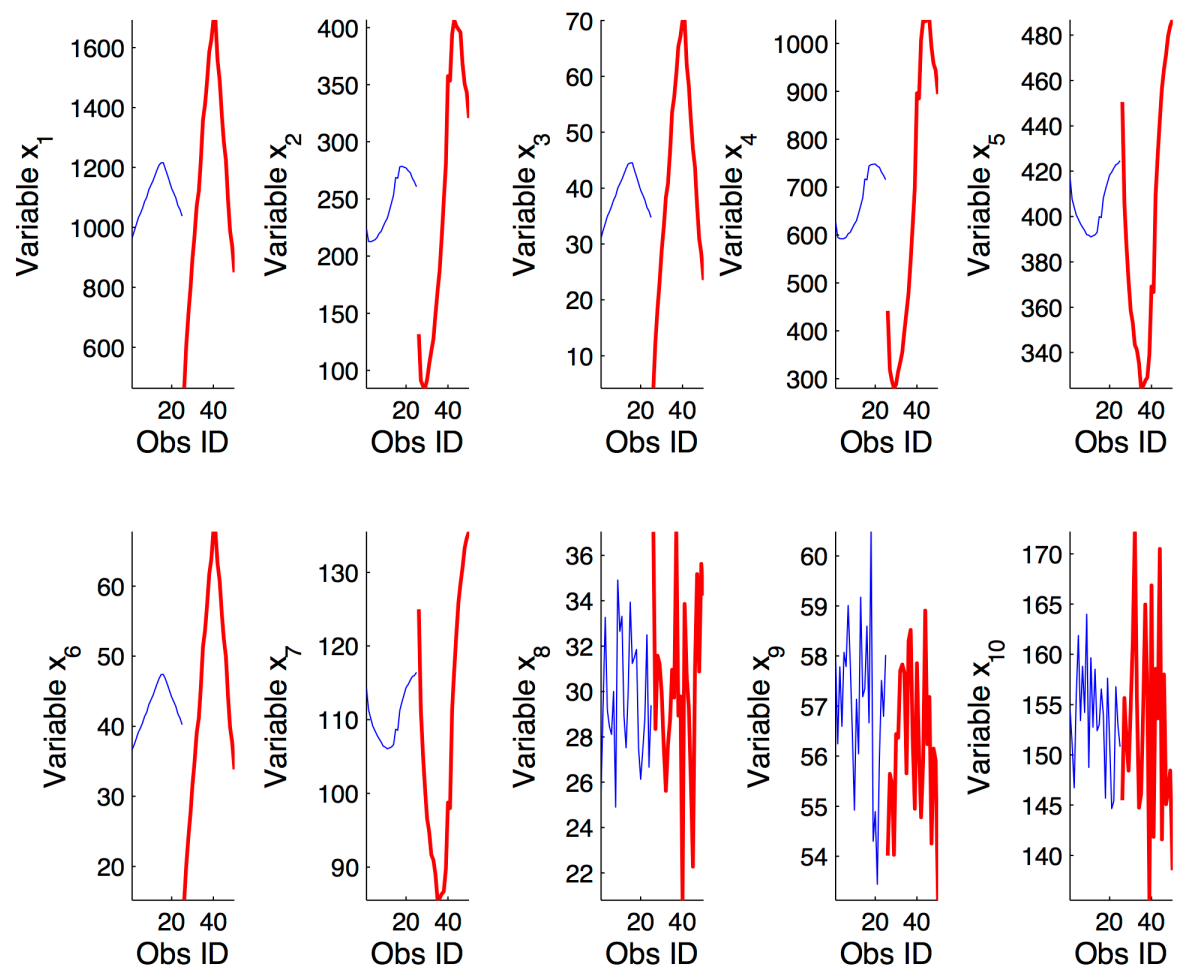

Figure 2: Temporal evolution of the variables of the simulated dataset for an on-specification (blue thin line) and an off-specification (red thick line) batch of the training set. 
a)

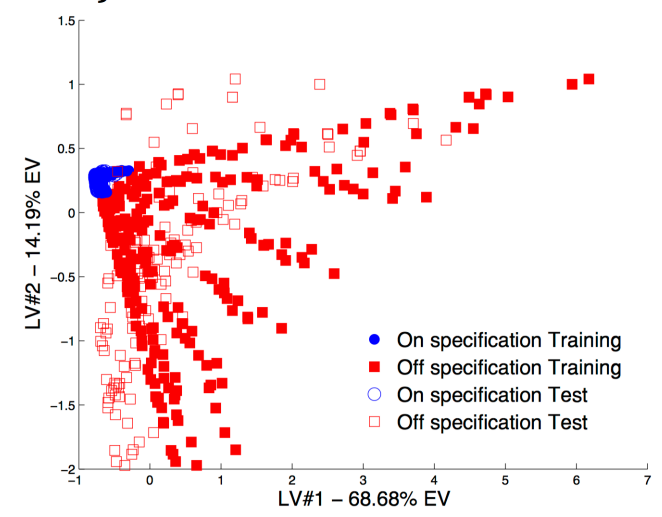

b)

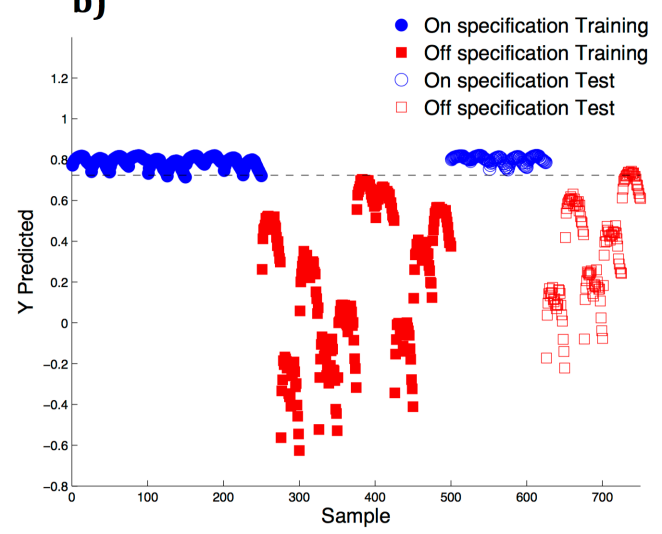

Figure 3: a) PLSDA scores plot of the $2^{\text {nd }}$-order polynomial kernel model built on the simulated data matrix and b) predicted $y$ values for both training and test sets. The black dotted line represents the probability threshold, calculated according to the Bayes' theorem [25]. (EV: Explained Variance). 


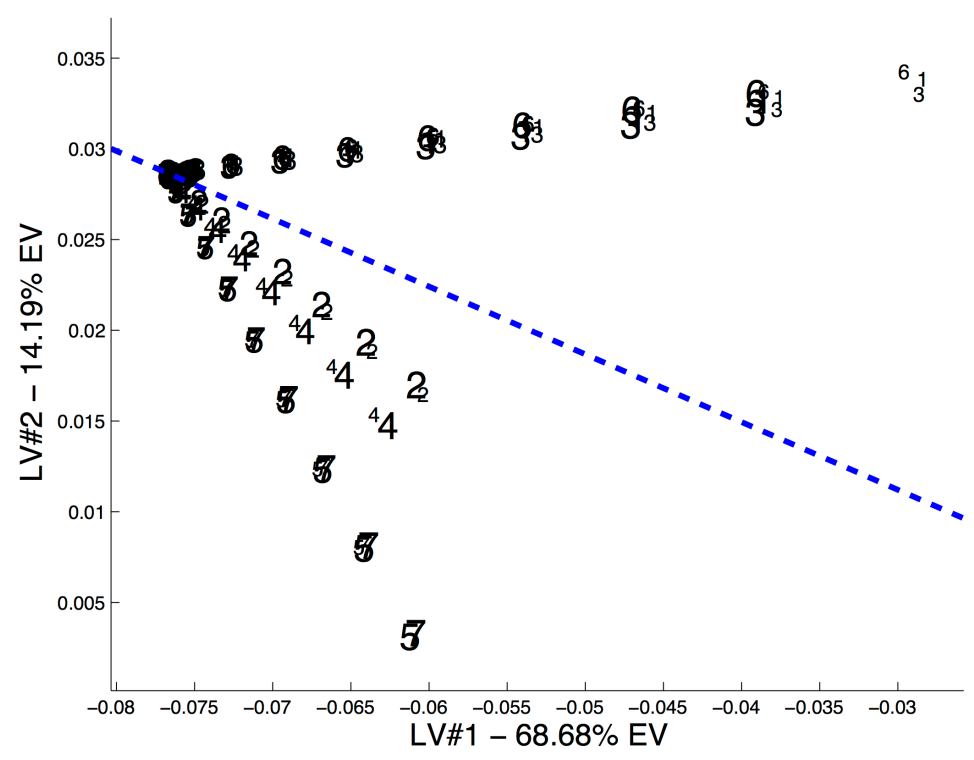

Figure 4: Pseudo-sample predicted scores plot for the $2^{\text {nd }}$-order polynomial kernel model built on the simulated dataset. The blue dotted line represents the discriminant direction between the centers of gravity of the two considered classes. (EV: Explained Variance). 
a)

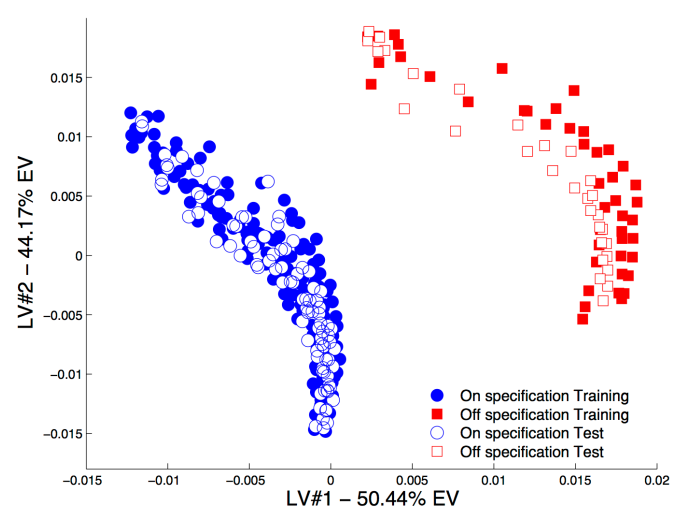

b)

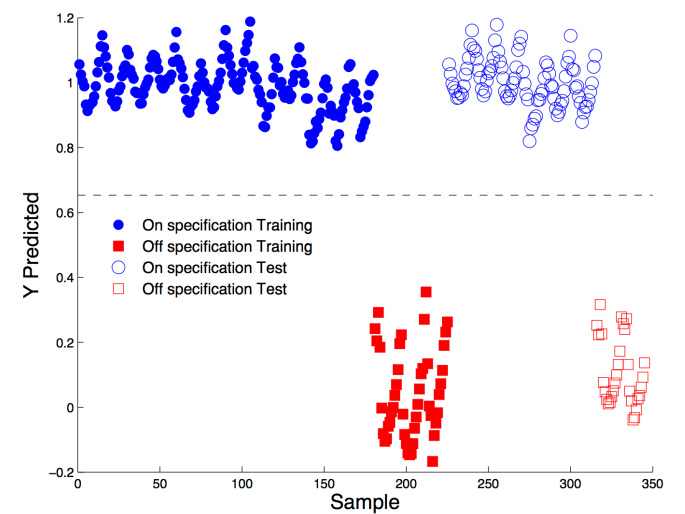

Figure 5: a) PLSDA scores plot of the model built on the reduced VWU kernel data matrix and b) predicted $y$ values for both training and test sets. The black dotted line represents the probability threshold, calculated according to the Bayes' theorem [25]. (EV: Explained Variance). Its use is justified by verifying that the response values calculated by the model for the observations of the training set are normally distributed within each single class. 


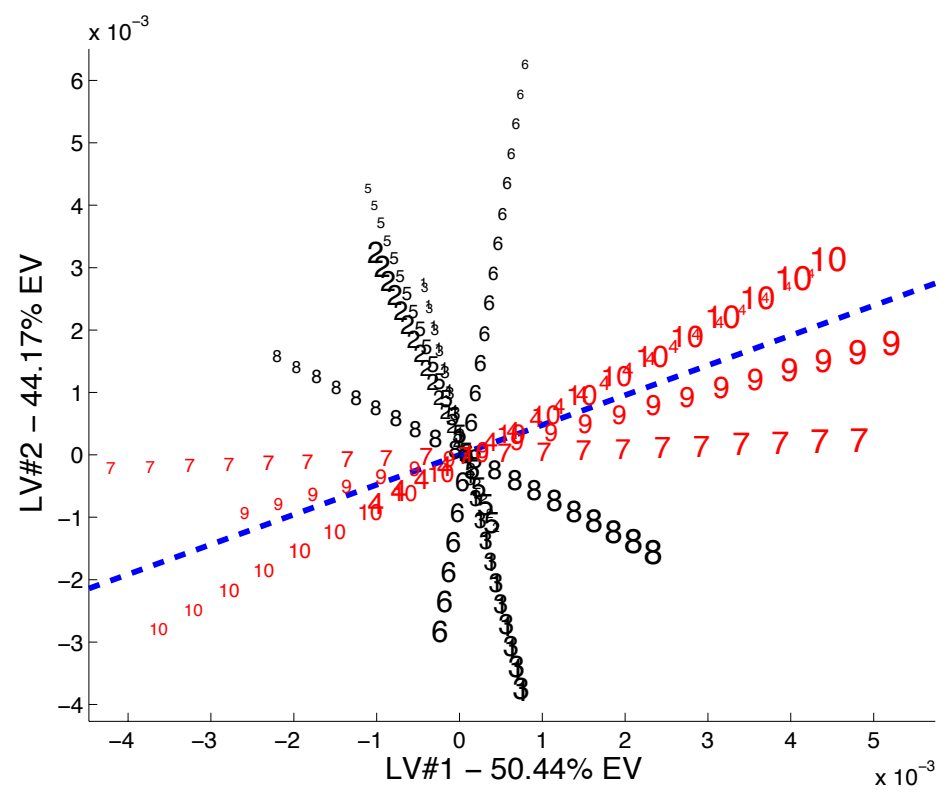

Figure 6: Pseudo-sample predicted scores plot for the reduced VWU kernel matrix. The blue dotted line represents the discriminant direction between the centers of gravity of the two considered classes. (EV: Explained Variance). 
a)

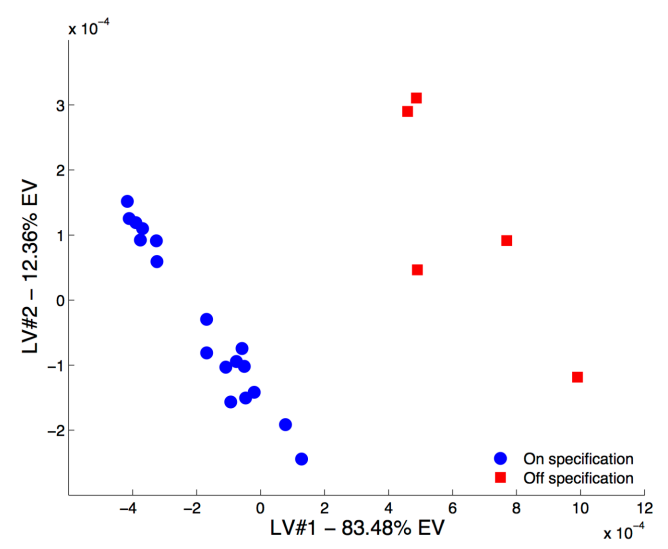

b)

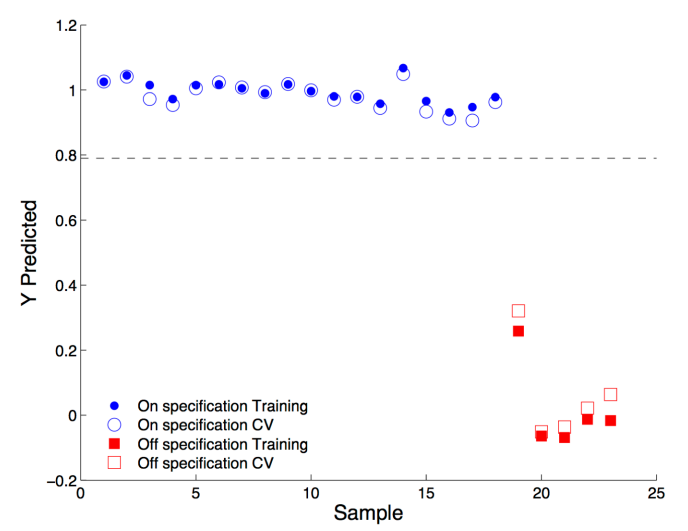

Figure 7: a) PLSDA scores plot of the model built on the BWU data matrix and b) predicted $y$ values for both training set and cross-validation. The black dotted line represents the probability threshold, calculated according to the Bayes' theorem [25]. (EV: Explained Variance). 

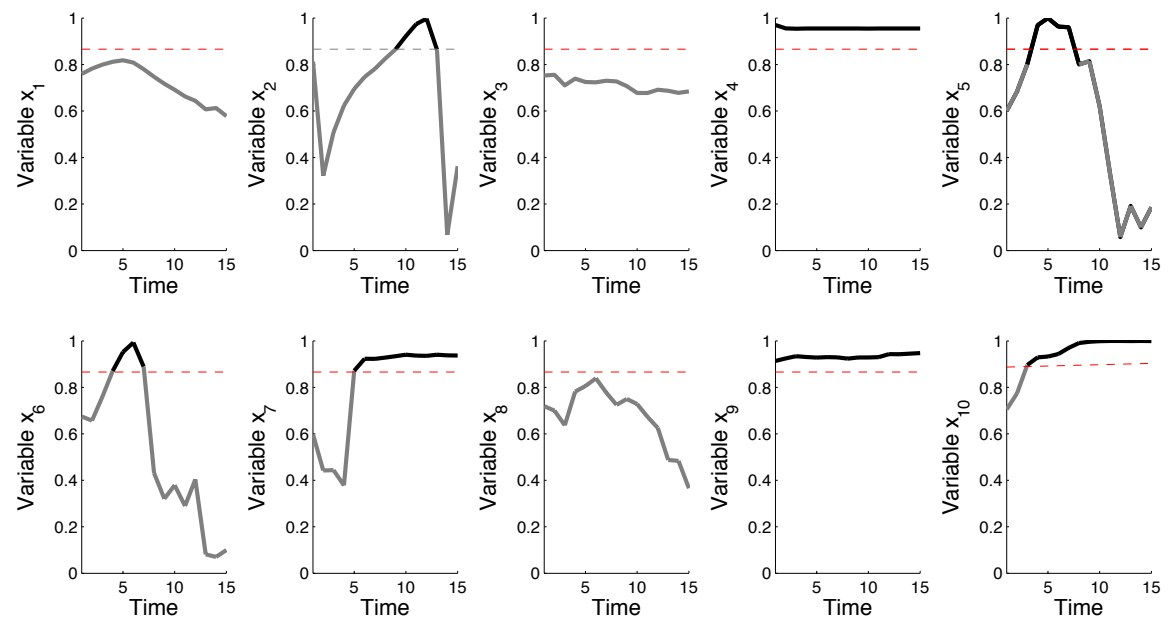

Figure 8: Values of the cosine of the angles formed by the pseudo-sample trajectories, related to each one of the original variables, and the discriminant direction at the different time points under study. The red dotted line represents the reference value of the cosine of a $30^{\circ}$ angle. 
a)

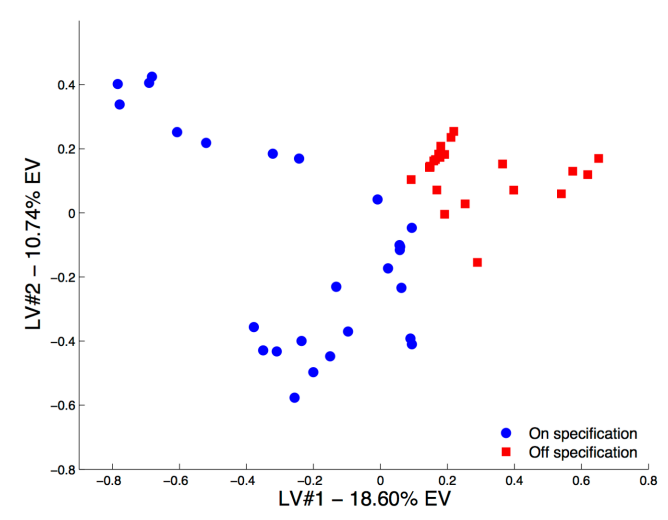

b)

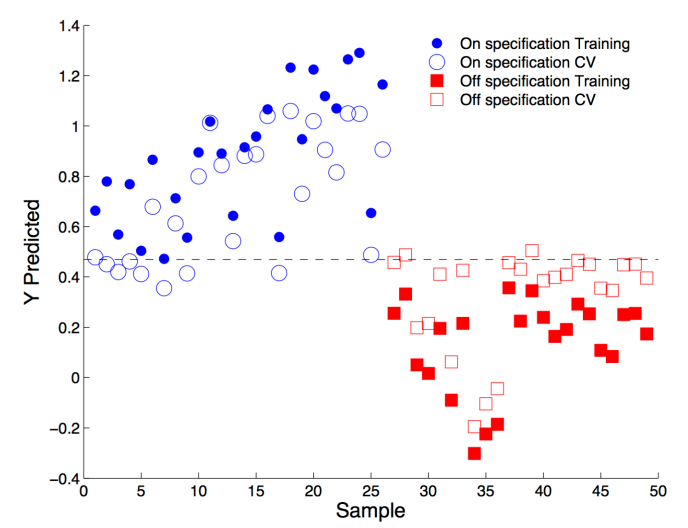

Figure 9: a) PLSDA scores plot of the model built on the LFE kernel data matrix and b) predicted $y$ values for both training set and cross-validation. The black dotted line represents the probability threshold, calculated according to the Bayes' theorem [25]. (EV: Explained Variance). 

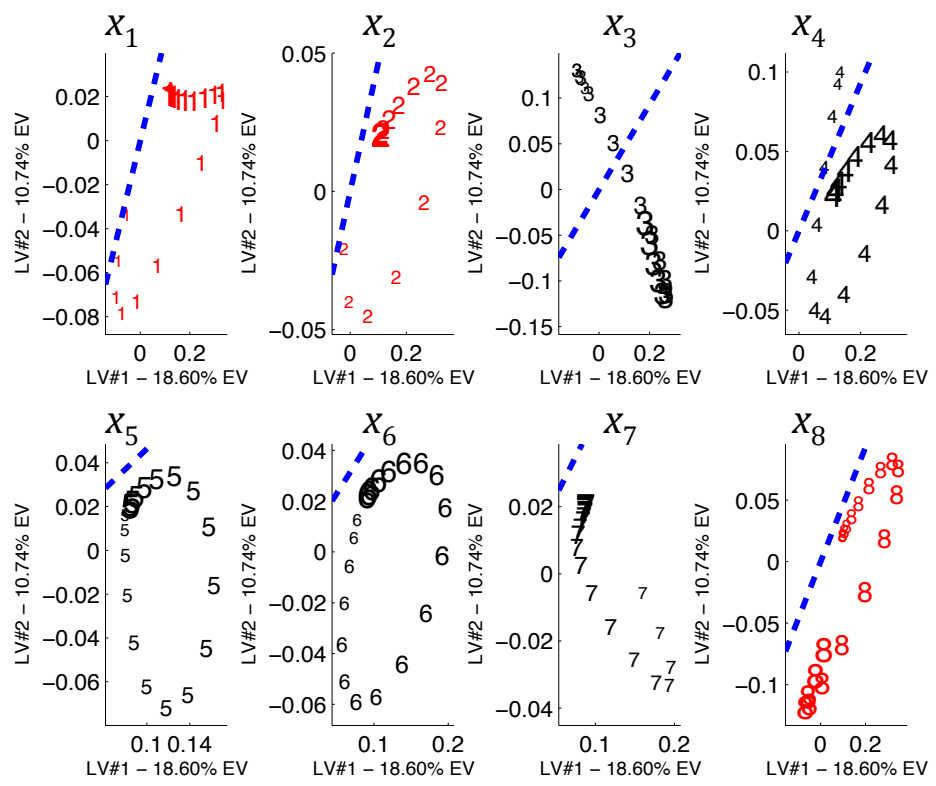

Figure 10: Pseudo-samples predicted scores plot for the LFE kernel matrix.

Each subplot contains a pseudo-sample trajectory for a specific variable. The blue dotted line represents the discriminant direction between the centers of gravity of the two considered classes. (EV: Explained Variance). 
a)

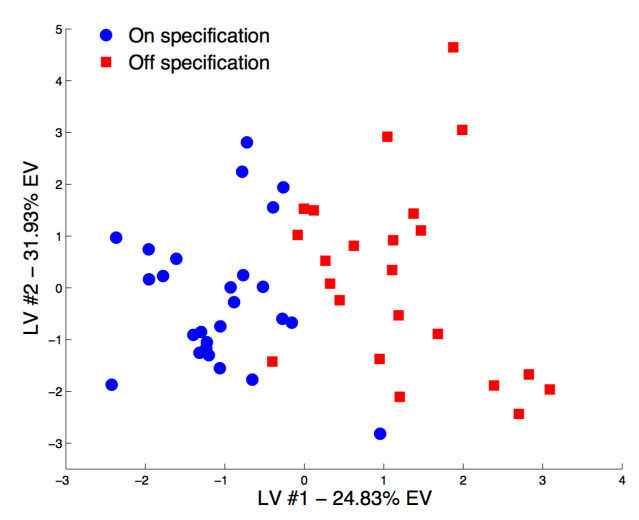

b)

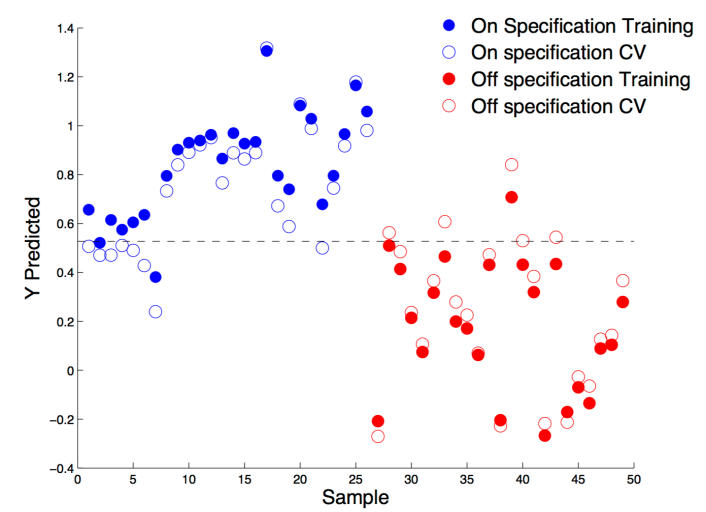

Figure 11: a) Scores and b) $y$-predicted plots obtained building a classical PLSDA model on the original LFE data matrix. The black dotted line represents the probability threshold, calculated according to the Bayes' theorem [25]. Correct classification rate in CV: $69.2 \%$ (on-specification), $78.3 \%$ (off-specification). (EV: Explained Variance) 
Table 1: Kernel functions used in this article and list of their adjustable parameters.

\begin{tabular}{ccc}
\hline Kernel Type & Kernel Function & Adjustable parameters \\
\hline Linear & $\mathbf{x}_{i}{ }^{\mathrm{T}} \mathbf{x}_{j}$ & None \\
2 $^{\text {nd_order polynomial }}$ & $\left(\mathbf{x}_{i}{ }^{\mathrm{T}} \mathbf{x}_{j}\right)^{2}$ & None \\
Gaussian & \\
$\exp \left(-\frac{\left\|\mathbf{x}_{i}-\mathbf{x}_{j}\right\|^{2}}{2 \sigma}\right)$ & $\sigma$ \\
\hline
\end{tabular}


Table 2: Latent variable number and correct classification rate of the 4 models built on the simulated dataset

\begin{tabular}{|c|c|c|c|c|c|}
\hline & \multirow[b]{2}{*}{ LV } & \multicolumn{4}{|c|}{ Correct classification rate $(\%)$} \\
\hline & & $\begin{array}{l}\text { Training } \\
\text { I class }\end{array}$ & $\begin{array}{l}\text { Training } \\
\text { II class }\end{array}$ & $\begin{array}{l}\text { Test } \\
\text { I class }\end{array}$ & $\begin{array}{c}\text { Test } \\
\text { II class }\end{array}$ \\
\hline PLSDA & 2 & 96.0 & 46.0 & 100 & 42.4 \\
\hline $\begin{array}{c}\text { K-PLSDA } \\
\text { (linear } \\
\text { kernel) }\end{array}$ & 2 & 95.6 & 45.2 & 100 & 42.4 \\
\hline $\begin{array}{c}\text { K-PLSDA } \\
\left(2^{\text {nd }} \text {-order }\right. \\
\text { polynomial } \\
\text { kernel })\end{array}$ & 2 & 98.4 & 100 & 100 & 92.8 \\
\hline $\begin{array}{c}\text { K-PLSDA } \\
\text { (rbf kernel, } \\
\sigma=0.5 \text { ) }\end{array}$ & 2 & 100 & 99.2 & 100 & 87.2 \\
\hline
\end{tabular}


Table 3: Values of the cosine of the angles formed by each pseudo-sample trajectory and the class discriminant direction (simulated data matrix).

\begin{tabular}{cccccccccc}
\hline Var. 1 & Var. 2 & Var. 3 & Var. 4 & Var.5 & Var.6 & Var. 7 & Var. 8 & Var. 9 & Var. 10 \\
\hline 0.88 & 0.97 & 0.89 & 0.92 & 0.80 & 0.87 & 0.81 & - & - & - \\
& & & & & & & & & \\
\hline
\end{tabular}


Table 4: Values of the cosine of the angles formed by each pseudo-sample trajectory and the class discriminant direction (VWU data matrix).

\begin{tabular}{llllllllll}
\hline Var. 1 & Var. 2 & Var. 3 & Var. 4 & Var.5 & Var.6 & Var. 7 & Var. 8 & Var. 9 & Var. 10 \\
\hline 0.27 & 0.11 & 0.27 & 0.99 & 0.18 & 0.53 & 0.92 & 0.49 & 0.99 & 0.98 \\
\hline
\end{tabular}

